\title{
Bulimia nervosa in obese patients qualified for bariatric surgery - clinical picture, background and treatment
}

\author{
Marzena Sekuła ${ }^{1,2}$, Iwona Boniecka³ ${ }^{3}$ Krzysztof Paśnik ${ }^{2}$ \\ ${ }^{1}$ University of Social Sciences and Humanities, University of Social Psychology, Warsaw, Poland \\ ${ }^{2}$ Department of General, Oncological, Metabolic and Thoracic Surgery, Military Institute of Medicine, Warsaw, Poland \\ ${ }^{3}$ Department of Clinical Dietetics, Medical University of Warsaw, Warsaw, Poland
}

Videosurgery Miniinv 2019; 14 (3): 408-414

DOI: https://doi.org/10.5114/wiitm.2019.81312

\begin{abstract}
Eating is a basic human physiological need which is necessary to keep the body alive. Eating disorders are diagnosed when eating (or not eating) and associated body weight gain anxiety become the main interest of an individual and all other spheres of life depend on it. Bulimia nervosa is a psychiatric disorder which is more and more commonly diagnosed in patients suffering from obesity and in patients after surgical treatment of obesity. In patients eligible for bariatric surgery this disorder should be diagnosed appropriately early and treated successfully before the procedure, because bulimia nervosa does not regress spontaneously. When untreated, it may last for years, reducing the efficacy of a surgical treatment of obesity, or even lead to complications that are health-and life-threatening for patients.
\end{abstract}

Key words: extreme obesity, bariatric surgery, bulimia nervosa.

\section{Introduction}

Obesity is a condition characterized by an amount of the adipose tissue which exceeds physiological reference ranges. According to the World Health Organization (WHO), obesity is the most dangerous chronic disease that does not tend to regress spontaneously. Therefore, it should be treated [1]. Bariatric procedures are currently considered to be the best method used for body mass reduction in patients with extreme obesity [2-4]. Long-term results show that their efficacy reaches $80 \%$, whereas for conservative treatment this rate is only approximately $5 \%$ [5]. Subjects with body mass index $(\mathrm{BMI}) \geq 40 \mathrm{~kg} / \mathrm{m}^{2}$ or $35 \mathrm{~kg} / \mathrm{m}^{2}$ and coexisting complications of obesity may be considered eligible for bariatric surgery $[4,6]$.

Surgical treatment without introducing lifestyle changes, including dietary habits, is known not to be associated with expected results [4, 7-15]. Studies showed that as many as $50-59 \%$ of bariatric surgery patients experienced weight regain to some extent [12], which may be connected with psychological factors, including concomitant nutritional disorders [8-14]. The severity of psychological disorders was related to the degree of the obesity, with a positive association found between the presence of psychopathology and the BMI [9].

Diagnostic criteria for bulimia nervosa are the same before or after bariatric surgery [16]. Research showed that diagnostic criteria for bulimia nervosa are met by approximately $2-49 \%$ of patients considered eligible for surgical treatment of obesity and by $7 \%$ to $22 \%$ of subjects after such treatment [17].

When untreated, bulimia may significantly affect the outcomes of the surgical treatment of obesity and general health status of patients [13]. Treatment of 
this condition should be considered a priority in subjects with coexisting obesity and bulimia, followed by treatment of excessive body weight [8]. This is particularly important due to the constantly growing number of bariatric operations in Poland [18].

\section{Diagnostic criteria for bulimia nervosa}

Bulimia nervosa is diagnosed based on diagnostic criteria. According to the guidelines of the American Psychiatric Association (DSM V) [16] these are:

1. Recurring episodes of binge eating over a short period of approximately $2 \mathrm{~h}$ when a patient consumes an amount of food that is greater compared to what others might eat in a similar period and similar circumstances.

2. These episodes are characterized by a lack of control over eating.

3. In order to avoid body weight increase, compensatory behaviors occur: inducing vomiting, using laxatives or dehydrating agents, or performing intensive physical exercises.

4. Individual self-esteem is significantly affected by body weight and shape.

5. The disorder is not only observed during episodes of anorexia nervosa.

6. Episodes of binge eating and compensatory behaviors are observed at least once a week over a period of 3 months [16].

\section{Etiology of bulimia nervosa}

The etiology of bulimia nervosa is complex and not completely clear. A combination of factors has to be present for this disease to develop. The reasons include the following: biological (genetic predisposition, impaired mechanism of satiety control, neurochemical disorders including serotonin deficiency), behavioral (abnormal eating habits and irregular eating, using nutritional restrictions), psychological (depression, impaired emotion regulation, low self-esteem with high expectations of oneself), systemic/family (impaired relations and communication between family members) and sociocultural (the cult of the body and aspiration to have a perfect body) conditions [19-21].

\section{Clinical picture of bulimia nervosa}

A consistent clinical picture of bulimia nervosa is observed only in a small group of patients. The phenomenon of diagnostic migration, occurring in the majority of patients, means the movement between diagnoses associated with eating disorders. Migration reflects a pattern in which eating disorders start with dietetic limitations, followed by failure of the self-control mechanism and leading to binge eating [22]. An episode of binge eating may be a single 2-hour episode that ends with compensatory behaviors, but it may also include a series of episodes interrupted with instances of behaviors aimed at eliminating food from the body that are typical of a given subject. Episodes of overeating and engaging in compensatory mechanisms constitute a method to indicate an approach to oneself and others, and a strategy of coping with affect, both positive and negative [23]. In subjects with an impaired process of emotion regulation, eating disorders may be pathologic and associated with an inappropriate amount, quality and frequency of food consumption [24].

\section{Psychopathology of bulimia nervosa}

Eating disorders have a common, specific psychopathological background that is cognitive and involves paying too much attention to body weight and possibilities of its control [22]. Patients suffering from bulimia nervosa base their self-esteem on their external appearance and not on their achievements and activities in various spheres of life. Additionally, they are characterized by dysfunctions in emotion interpretation and regulation (the lack of ability to cope with difficult emotions), which leads to an increased intensity of episodes of binge eating and purging as a method of releasing emotional tension (Figure 1). Bulimia nervosa is often a way to reduce

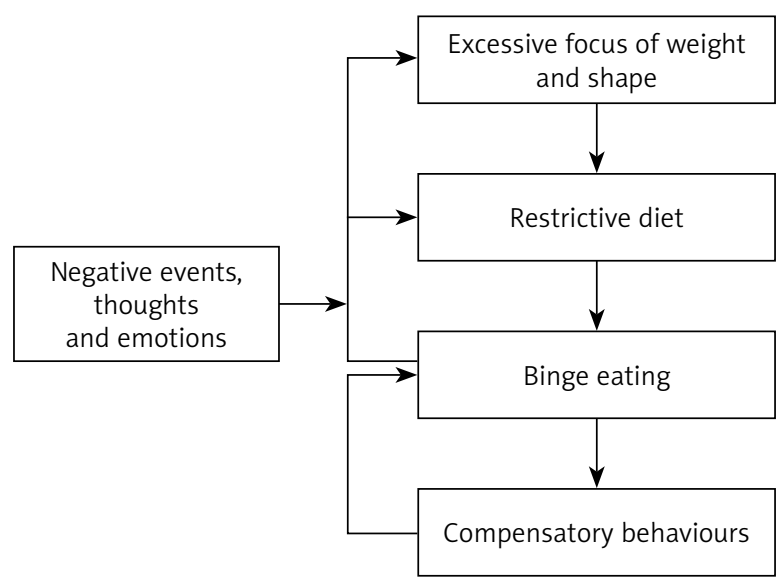

Figure 1. Bulimia nervosa development [23] 
excessive body weight and to maintain a slim fig ure. Episodes of overeating and compensatory behavior caused by "bulimic drive for overeating" [25] result in the dysregulation of body homeostasis and mechanisms responsible for hunger and satiety, as well as an impaired interpretation of these signals. Therefore, a vicious cycle of binge eating is initiated, followed by strict dietary restrictions and their emotional consequences. Compensatory behaviors affect the functioning of the endocrine system, resulting in changes of the drive to eat, depressed mood, an increased feeling of hunger and increased frequency of binge eating and compensatory behaviors [26]. This process leads to the so-called "bulimic trap" $[23,26]$.

\section{Health and psychological consequences of bulimia nervosa}

When untreated, this disease affects the functioning of the whole body. Somatic and psychological consequences are present from the onset. Somatic complications significantly depend on individual compensatory behaviors. The most common chemical effects exerted by gastric content on the esophagus and oral cavity include: enamel erosion and discoloration accompanied by dental caries, gingival diseases, inflammatory edema of parotid glands, erosions of the esophagus and the posterior wall of the throat, hoarse voice, pain within the facial nerve, and tinnitus. Vomiting, using laxatives and dehydrating agents may also favor disturbances of water and electrolyte balance, and acid and alkaline balance. Inducing vomiting may result in the following consequences: metabolic alkalosis with hyperchloremia, increased levels of carbohydrates, secondary hypokalemia, and hypovolemia with secondary hyperaldosteronism. The presence of vomiting and diarrhea, and the use of diuretics, may lead to metabolic acidosis with apparently normal potassium levels, hypokalemic nephropathy with disorders of urine concentration, hypochloremic alkalosis, and hypomagnesemia. Moreover, dorsal surfaces of the hands are injured because of inducing vomiting. Other consequences include gastric erosions, pancreatitis, gastroesophageal reflux disease, menstruation disorders and swelling of lower extremities, dyspnea, heart rhythm disturbances, reduced arterial pressure, reduced ejection fraction, poorly palpable heart rate, poorly audible heart sounds, abdominal distention, paralytic ileus, neuropathies, increased thirst, myopathy, seizures, muscle contractions and avitaminosis [27, 28].

\section{Health consequences of bulimia in bariatric surgery patients}

Water and electrolyte imbalance, acid and alkaline imbalance and esophageal injuries are especially dangerous in the postoperative period in obese patients in whom bulimia has not been treated before bariatric surgery. Esophageal injuries, if present for a longer period, may lead to erosions, dangerous bleeding or even esophageal rupture (Boerhaave syndrome) [28]. Deficiencies associated with the surgery alone, such as deficiencies of vitamins, minerals or protein $[29,30]$, may progress due to the chronic use of laxatives, dehydrating agents and inducing vomiting. As gastroesophageal reflux disease is often observed in obese patients and its symptoms may also accompany bulimia nervosa, such patients have to be carefully monitored. It is well known that chronic esophageal reflux may favor the development of Barrett's esophagus, which is a precancerous condition involving intestinal metaplasia of the esophageal epithelium [28, 31, 32].

Episodes of overeating may also lead to dumping syndrome (DS) in these patients. It is usually observed after partial or complete gastric resection, especially after Roux-Y-en gastric bypass (RYGB) bariatric surgery $[29,33]$. However, according to the latest studies it may also develop after sleeve gastrectomy (SG) $[34,35]$. The RYGB and SG are the two most common bariatric procedures performed in Poland and worldwide [18]. Early and late DS may be distinguished depending on the period which has passed since a meal was consumed. Early symptoms are observed within $1 \mathrm{~h}$ after a meal $[34,35]$, usually after 10-30 $\min$ [36], and result from the rapid passage of food from the stomach to the intestines because of reduced gastric volume or faster gastric emptying [34]. Consequently, food content with high osmolality that is especially rich in simple carbohydrates and fats is quickly introduced into the intestines. Therefore, water is relocated from blood vessels into the intestinal lumen $[33,37]$. It leads to the development of gastrointestinal complaints (abdominal pain, diarrhea, flatulence, feeling of fullness, nausea, vomiting), as well as vasculomotor complaints (fatigue, facial erythema, palpitations, cold 
sweats, tachycardia, dizziness, headache, fainting) Late symptoms are usually observed within 1-3 h after a meal and are manifested as reactive hypoglycemia [33-37] that results from excessive insulin secretion [33]. They include hyperhidrosis, palpitations, hunger, fatigue, problems with concentration, disorientation, aggression, trembling and fainting $[33,35,36]$. In the case of hypoglycemia, hospitalization may sometimes be necessary [36].

Apart from somatic effects, bulimia nervosa also has psychological consequences. Patients are excessively focused on eating, which becomes their main interest. They feel obliged to hide the disease because of engaging in compensatory mechanisms. Therefore, they withdraw from interpersonal contacts. A pathologic sense of guilt and associated tension occur in the majority of patients [27].

\section{Prognosis in bulimia nervosa}

Prognosis regarding the treatment of patients with bulimia nervosa is better with early diagnosis and introduction of treatment. According to the literature the rates of treatment improvement range between $30 \%$ and $60 \%$ [38, 39].

\section{Individualized therapy}

The treatment of patients with obesity and bulimia nervosa should be comprehensive. The team of specialists should include a psychologist-psychotherapist or a psychiatrist. The aim of such treatment includes not only the resumption of normal dietary habits, but mostly the change in the mental functioning of the individual.

Early diagnosis of the disorder facilitates earlier provision of specialist psychological and psychiatric care to such patients. Therefore, it favours improvement of the prognosis and the effectiveness of bariatric treatment. Making the diagnosis is not easy, because the patients are commonly concerned about a psychological assessment as they had never dealt with a mental health specialist or due to a fear of being disqualified from bariatric surgery. It is necessary for the patient to understand the importance of the treatment, be open to cooperate and accept assistance. A nutritional disorder concomitant with obesity does not disqualify the patient from bariatric surgery. The patient should start treating bulimia prior to the bariatric procedure. If the disorder is untreated it will not resolve with surgery, but it may contribute to health status deterioration and affect long-term treatment outcomes. Therefore, establishing rapport based on the feeling of confidence and security and cooperation between a psychologist and the patient is crucial in effective treatment [40].

Regrettably, no clear guidelines are available which describe mental status assessment in such cases. The diagnosis of bulimia nervosa is based on detailed history and observation of the patient. Meeting ICD-10 or DSM-V diagnostic criteria is necessary for the disorder to be diagnosed by a psychologist (psychological diagnosis) or a psychiatrist (nosological diagnosis) [16, 41].

Psychologists may use diagnostic tools depending upon actual needs, a therapeutic goal and in order to confirm the diagnosis. Regrettably, few tools have been adapted to Polish conditions. The Questionnaire of Eating Behavior authored by OgińskaBulik and Putyński is one of those tools [42]. It helps diagnose nutritional disorders, predict a tendency towards weight gain and is used during the selection of intervention and treatment. Additionally, a psychologist may use other screening tools which are employed for early diagnosis of nutritional disorders: the EDI questionnaire [43] and its updated versions - SCOFF questionnaire [44], the ESP questionnaire [45] or the EAT-26 questionnaire [46]. It needs to be remembered that clinical nomenclature describes a real human and his suffering due to experiencing a nutritional disorder. Therefore, treatment effectiveness requires concentrating on the psychopathology of a nutritional disorder in the first place and then on the preparation of the patient for the surgical treatment of obesity.

The process of treating a patient with bulimia nervosa and obesity may be divided into 5 stages [22]. Stage I encompasses psychoeducation and strengthening the patient's motivation for treatment. At this stage it is most important to establish rapport with the patient and select common and clear goals of the treatment. The patient's willingness to start treatment does not explicitly mean meeting a psychologist or a dietitian. Preparation and appropriate motivation for treatment is a process which occurs according to specific stages of changes, which is best pictured by the transtheoretical model of change [47].

Stage II consists in the stabilization of the physical status of the patient, normalization of nutritional behaviors and elimination of destructive compen- 
satory behaviors through control of dietary habits and training of dealing with emotions in difficult situations. Stage III concentrates on the core of psychopathology and cognitive restructuring. It involves the change of abnormal regimens and beliefs concerning eating and body weight, and improving interpersonal relationships. Stage IV encompasses the prevention of bulimia nervosa recurrences, predicting difficult situations which promote a relapse of manifestations. Stage $V$ is the process of preparing the patient for surgical obesity treatment [22].

\section{Treatment of bulimia nervosa in obese patients subject to bariatric surgery}

According to recommendations concerning surgical obesity treatment, the decision to qualify a patient for a bariatric surgery should be made by a team of specialists including a surgeon, a dietitian and a psychologist. Moreover, in some cases a psychiatric consultation is also recommended [6]. The patient needs to be informed about negative consequences resulting from ignoring dietary recommendations and engaging in compensatory behaviors aiming to control excessive body weight [8]. The patient should be aware that compensatory behaviors are ineffective, because food consumed during an episode of overeating becomes partially digested before laxatives start working or vomiting is induced [22]. Compensatory vomiting is a behavior that may be observed after bariatric surgery as a consequence of incompliance with postoperative dietetic recommendations. In a group of patients with bulimia nervosa who are qualified for surgical treatment of obesity this complication seems to be especially dangerous, as it favors the development of postoperative complications and disease recurrence. According to Yashkov and Bekuzarov [14], frequent vomiting typical of patients incompliant with dietetic recommendations, especially after typical restrictive surgery, may be treated as a symptom of so-called iatrogenic bulimia. Interestingly, according to these authors, bulimia nervosa which is severe and resistant to treatment may be considered as a latent and especially dangerous form of extreme obesity [14].

With regard to patients qualified for bariatric surgery, bulimia nervosa has to be treated first, as mentioned above. However, it seems that even in patients receiving psychological therapy, a specific surgical procedure should be considered because of a high risk of recurrences. Considering the fact that gastric juice irritates the esophageal mucosa due to gastroesophageal reflux (which accompanies both obesity and bulimia nervosa) or inducing vomiting (in the course of compensatory behaviors), it is usually recommended to perform gastrointestinal bypass procedures in this group of patients. Such a procedure is recommended by the majority of specialists in the group of patients with gastroesophageal reflux disease [32, 48-50], especially a severe form, mainly in patients with Barrett's esophagus [32, 48, 49, 51, 52]. Although sleeve gastrectomy is a popular procedure, resulting in significant body weight loss due to a restrictive mechanism (limited gastric volume) and a hormonal mechanism (reduced ghrelin production), it is generally not recommended in such cases because gastric juice has a higher $\mathrm{pH}$ compared to RYGB and factors such as reduced pressure of the lower esophageal sphincter increase the tendency for gastroesophageal reflux disease [51]. According to Braghetto and Csendes [48, 49], Barrett's esophagus may be a late complication of this procedure. Therefore, this procedure should be avoided in patients from the risk group, including those with bulimia nervosa. In obese patients with bulimia, especially bulimia that is resistant to conservative treatment, Yashkov and Bekuzarov [14] recommended procedures such as biliopancreatic diversion or biliopancreatic diversion with a duodenal switch. The authors claimed that such procedures result in hormonal changes despite the fact that in the late postoperative period they do not limit the amount of consumed food. Consequently, it results in appetite inhibition, and, moreover, reduction in fat absorption [14]. However, one should remember that these procedures may cause great deficiencies leading to other metabolic diseases [29].

Lack of understanding of the process, lack of cooperation between the patient and the therapeutic team, and inability to participate in permanent longterm multispecialist follow-up after the surgery are absolute contraindications for surgical obesity treatment [6].

\section{Conclusions}

Bulimia nervosa is a mental condition that is commonly concomitant with obesity [8-14]. When untreated, it triggers serious psychological, social and health consequences. 
Treatment should focus on the effective diagnosis and treatment at first, and then on the reduction of excessive body weight. Treatment should be comprehensive because of the complex etiology of bulimia and obesity, and their mutual relations. Therapy should include lifestyle changes, including eating habits, as well as improvement of the mental functioning of an individual, as it increases the chances of effective treatment. Therefore, cooperation is necessary between a patient and a multi-specialist team including a surgeon, psychologist, psychiatrist, and dietician.

Mental disorders, including bulimia nervosa, are not exclusion criteria for a bariatric surgery, but in order to provide optimal outcomes it is important to identify and treat all the elements favoring increased body weight before a surgical intervention $[8,13]$.

According to Yashkov and Bekuzarov [14], all candidates for surgical treatment of obesity should be assessed for any eating disorders, as they may affect the efficacy and selection of a surgical procedure. In the case of patients qualified for bariatric surgery, bulimia nervosa may be an indication for a gastric bypass procedure.

It is necessary to establish clear and transparent guidelines regarding psychological assessment of patients with eating disorders who are considered eligible for bariatric surgery.

\section{Conflict of interest}

The authors declare no conflict of interest.

\section{References}

1. Global status report on noncommunicable diseases 2014. "Attaining the nine global noncommunicable diseases targets; a shared responsibility". World Health Organization, Geneva 2014.

2. Sekuła M, Paśnik K. Bariatric surgery effectively fights obesity [Polish]. Lek Wojsk 2016; 94: 347-51.

3. Sekuła M, Paśnik K. Evaluation of the sleeve gastrectomy effectiveness in the treatment of morbid obesity [Polish]. Lek Wojsk 2017; 95: 64-7.

4. Fried M, Yumuk V, Oppert JM, et al. Interdisciplinary European guidelines on metabolic and bariatric surgery. Obes Surg 2014; 24: 42-55.

5. Paśnik K, Najdecki M, Koziarski T, et al. New trends in bariatric surgery [Polish]. Pol Merk Lek 2009; 26: 539-41.

6. Budzyński A, Major P, Głuszek S, et al. Polish recommendations on bariatric and metabolic surgery [Polish]. Med Prakt Chir 2016; 6: 13-26.
7. Boniecka I, Szczygieł B, Paśnik K. Selected lifestyle characteristic of patients with extreme obesity qualified for bariatric surgery [Polish]. Roczn PZH 2009; 60: 279-84.

8. Gallé F, Maida P, Cirella A, et al. Does post-operative psychotherapy contribute to improved comorbidities in bariatric patients with borderline personality disorder traits and bulimia tendencies? A prospective study. Obes Surg 2017; 27: 1872-8.

9. Abilés V, Rodríguez-Ruiz S, Abilés J, et al. Psychological characteristics of morbidly obese candidates for bariatric surgery. Obes Surg 2010; 20: 161-7.

10. Van Hout GCM, Verschure SKM, van Heck GL. Psychosocial predictors of success following bariatric surgery. Obes Surg 2005; 15: 552-60.

11. de Zwaan M, Hilbert A, Swan-Kremeier L, et al. Comprehensive interview assessment of eating behavior 18-35 months after gastric bypass surgery for morbid obesity. Surg Obes Relat Dis 2010; 6: 79-85.

12. Ivezaj V, Kessler EE, Lydecker JA, et al. Loss-of-control eating following sleeve gastrectomy surgery. Surg Obes Relat Dis 2017; 13: 392-8.

13. Taylor VH, Sharma AM. A patient with personal control of the adjustable gastric band and bulimia: a psychiatric complication. Obes Surg 2006; 16: 1386-7.

14. Yashkov Y, Bekuzarov DK. Effectiveness of biliopancreatic diversion in the patients with bulimia nervosa. Obes Surg 2006; 16 : 1433-9.

15. Boniecka I, Wileńska H, Jeznach-Steinhagen A, et al. Stress as a factor contributing to obesity in patients qualified for bariatric surgery - studies in a selected group of patients (a pilot study). Videosurgery Miniinv 2017; 12: 60-7.

16. Gałecki P, Święcicki Ł. Diagnostic criteria from DSM V [Polish]. Edra Urban \& Partner, Wroclaw 2015.

17. Morseth MS, Hanvold SE, R $\varnothing$, et al. Self-reported eating disorder symptoms before and after gastric bypass and duodenal switch for super obesity a 5-year follow-up study. Obes Surg 2016; 26: 588-94.

18. Walędziak M, Różańska-Walędziak A, Kowalewski PK, et al. Present trends in bariatric surgery in Poland. Videosurgery Miniinv DOI: 10.5114/wiitm.2018.77707.

19. Fairburn CG, Welch SL, Doll HA, et al. Risk factors for bulimia nervosa: a community-based case-control study. Arch Gen Psychiatr 1997; 54: 509-17.

20. Bulik C. Significant linkage on chromosome 10p in families with bulimia nervosa. Am J Hum Gen 2009; 72: 200-7.

21. Pilecki MW. Psychogenic determinants of bulimia nervosa [Polish]. Przegl Lek 2009; 66: 106-9.

22. Fairburn CG. Cognitive-behavioral therapy and eating disorders [Polish]. WUJ, Krakow 2013.

23. Mroczkowska D, Ziółkowska B. Bulimia from A to Z [Polish]. Difin, Warsaw 2011.

24. Sekuła I, Boniecka I, Paśnik K. Assessment of health behaviors, nutritional behaviors, and self-efficacy in patients with morbid obesity. Psychiatr Pol Online first Nr 105: 1-13. DOI: https://doi. org/10.12740/PP/OnlineFirst/81182.

25. Jablow M. Anorexia, bulimia, obesity [Polish]. GWP, Gdansk 2000. 
26. Maine M, Hartman-McGilley B, Bunnell DW (eds.). Treatment of Eating Disorders. A Bridge between Science and Practice. Elsevier Urban \& Partner, Wroclaw 2010.

27. Ogden J. Psychology of nutrition. From healthy to disturbed eating behaviors [Polish]. WUJ, Krakow 2011.

28. Mehler PS, Rylander M. Bulimia nervosa - medical complications. J Eat Disord 2015; 3: 12.

29. Mechanick JI, Youdim A, Jones DB, et al. Clinical practice guidelines for the perioperative nutritional, metabolic, and nonsurgical support of the bariatric surgery patient--2013 update: cosponsored by American Association of Clinical Endocrinologists, the Obesity Society, and American Society for Metabolic \& Bariatric Surgery. Endocr Pract 2013; 19: 337-72.

30. Parrott J, Frank L, Rabena R, et al. American Society for Metabolic and Bariatric Surgery Integrated Health Nutritional Guidelines for the Surgical Weight Loss Patient 2016 Update: Micronutrients. Surg Obes Relat Dis 2017; 13: 727-41.

31. Varela JE. Barrett's esophagus: a late complication of laparoscopic adjustable gastric banding. Obes Surg 2010; 20: 244-6.

32. Felsenreich DM, Kefurt R, Schermann M, et al. Reflux, sleeve dilation, and Barrett's esophagus after laparoscopic sleeve gastrectomy: long-term follow-up. Obes Surg 2017; 27: 3092-101.

33. Chaves YD, Destefani AC. Pathophysiology, diagnosis and treatment of dumping syndrome and its relation to bariatric surgery. Arq Bras Cir Dig 2016; 29 (Suppl 1): S116-9.

34. Papamargaritis D, Koukoulis G, Sioka E, et al. Dumping symptoms and incidence of hypoglycaemia after provocation test at 6 and 12 months after laparoscopic sleeve gastrectomy. Obes Surg 2012; 22: 1600-6.

35. Tzovaras G, Papamargaritis D, Sioka E, et al. Symptoms suggestive of dumping syndrome after provocation in patients after laparoscopic sleeve gastrectomy. Obes Surg 2012; 22: 23-8.

36. Laurenius A, Olbers T, Näslund I, Karlsson J. Dumping syndrome following gastric bypass: validation of the dumping symptom rating scale. Obes Surg 2013; 23: 740-55.

37. Deitel $M$. The change in the dumping syndrome concept. Obes Surg 2008; 18: 1622-4.

38. Michtell LE, Pyle RL. A long-term follow-up study of outpatient with bulimia nervosa treated in a structured group psychotherapy program. In: The Course of heating disorders. Long-term Follow-up Studies of Anorexia Nervosa and Bulimia Nervosa. Herzog W, Deter HG, Vandereycken W (eds.). Springer, Berlin, Heidelberg 2016; 174-81.

39. Lacey JH. Long-term follow-up of bulimic patients treated in integrated behavioural and psychodynamic treatment programmes. In: The Course of heating disorders. Long-term Follow-up Studies of Anorexia Nervosa and Bulimia Nervosa. Herzog W, Deter HG, Vanndereycken W (eds). Springer, Berlin, Heidelberg 2016; 150-73.

40. Stemplewska-Żakowicz K. Psychological diagnosis [Polish]. GWP, Gdansk 2016.

41. Pużyński S, Wciórka J. Classification of mental disorders and behavioral disorders in ICD-10. Clinical descriptions and diagnostic guidelines [Polish]. Vesalius, Krakow 2000.

42. Ogińska-Bulik N, Putyński L. My Eating Habits Questionnaire - structure and psychometric properties [Polish]. Acta Universitatis Lodziensis. Fol Psych 2000; 4: 25-31.
43. Crowther JH, Lilly RS, Crawford PA, Shepherd KL. The stability of the eating Disorder Inventory. Int J Eat Disord 1992; 12: 97-101.

44. Morgan JF, Reid F, Lacey JH. The SCOFF questionnaire assessment of a new screening tool for eating disorders. BMJ 1999; 319: 1467-8.

45. Cotton MA, Ball C, Robinson P. Four simple questions can help screen for eating disorders. J Gen Intern Med 2003; 18: 53-6.

46. Mintz LB, O'Halloran MS. The Eating Attitudes Test: validation with DSM-IV eating disorder criteria. J Pers Assess 2000; 74: 489-503.

47. Miller WR, Rollnick S. Motivational dialogue. How to help people change [Polish]. WUJ, Krakow 2014.

48. Braghetto I, Csendes A. Patients having bariatric surgery: surgical options in morbidly obese patients with Barrett's esophagus. Obes Surg 2016; 26: 1622-6.

49. Braghetto I, Csendes A. Prevalence of Barrett's esophagus in bariatric patients undergoing sleeve gastrectomy. Obes Surg 2016; 26: 710-4.

50. Burgerhart JS, Schotborgh CA, Schoon EJ, et al. Effect of sleeve gastrectomy on gastroesophageal reflux. Obes Surg 2014; 24: 1436-41.

51. Kindel TL, Oleynikov D. The improvement of gastroesophageal reflux disease and Barrett's after bariatric surgery. Obes Surg 2016; 26: 718-20.

52. Gagner M. Is sleeve gastrectomy always an absolute contraindication in patients with Barrett's? Obes Surg 2016; 26: 715-7.

Received: 24.10.2018, accepted: 27.11.2018. 